\title{
Beaming of electromagnetic waves emitted through a subwavelength annular aperture
}

\author{
Humeyra Caglayan, Irfan Bulu, and Ekmel Ozbay \\ Department of Physics and the Nanotechnology Research Center, Bilkent University, 06800 Ankara, Turkey
}

Received July 5, 2005; accepted August 21, 2005; posted September 1, 2005 (Doc. ID 63177)

\begin{abstract}
We study the diffraction of electromagnetic waves from subwavelength metallic circular apertures in the microwave spectrum. The theoretical and experimental demonstration of the near- and far-field electromagnetic distributions for subwavelength circular annular apertures and circular annular apertures surrounded by concentric periodic grooves are reported here. The metallic samples had a subwavelength hole with a diameter of $8 \mathrm{~mm}$ and had concentric grooves with a periodicity of $16 \mathrm{~mm}$. We present the angular transmission distributions from circular annular apertures, and circular annular apertures surrounded by concentric periodic grooves. At the surface-mode resonance frequency the transmitted electromagnetic waves from the subwavelength circular annular aperture surrounded by concentric periodic grooves have a strong angular confinement with an angular divergence of $\pm 3^{\circ}$. This represents a fourfold reduction when compared with the angular divergence of the beam transmitted from the subwavelength circular aperture. () 2006 Optical Society of America
\end{abstract}

OCIS codes: $240.6680,230.1950$.

\section{INTRODUCTION}

The transmission of electromagnetic (EM) waves through a single subwavelength aperture has been studied for many years. As defined in the standard diffraction theory by Bethe ${ }^{1}$ in 1944, a circular aperture with a subwavelength diameter transmits EM waves very poorly $\sim(d / \lambda)^{4}$, and exiting EM waves are fully diffracted in all directions. This aperture had two disadvantages: low transmission as well as diffraction, the main problems of manipulating light, especially for subwavelength scales. However, it has been shown that not only the enhancement of the transmission but also the confinement of the transmitted beam is possible by means of surrounding the metal surface of the subwavelength apertures with periodic corrugations. ${ }^{2}$ These effects are attributed to the coupling of incident light to surface plasmons (SPs). This idea has stimulated studies on this subject, and numerous experimental and theoretical work has been performed in the optical and microwave regimes. ${ }^{3-11}$

SPs are the collective excitation of electrons at the surface of a conductor in the longitudinal direction. As SP modes have longer wave vectors than light waves of the same energy, EM radiation does not interact with the SP modes of a smooth metal surface. ${ }^{12}$ When the metal surface surrounding the subwavelength hole is corrugated, the incident light can couple to SPs. A resonant interaction leads to an enhanced transmission at wavelengths determined by the corrugation period. ${ }^{13,14}$

In 1992 optical near-field microscope researchers proposed the use of coaxial aperture to achieve enhanced transmission. ${ }^{15}$ Baida et al. ${ }^{16}$ proposed a similar idea, stating that it was possible to optimize the transmission enhancement and angular confinement using a subwavelength circular coaxial aperture with a surrounding array of grooves. Recently, we have reported enhanced transmission in microwave regime using structures with sub- wavelength circular coaxial apertures. This enhanced transmission is assisted by the guided mode of the coaxial waveguide and coupling to the surface plasmons. ${ }^{17}$ In this paper, we report the near-field and far-field distributions of EM waves emitted from subwavelength circular annular apertures and circular annular apertures surrounded by concentric periodic grooves.

\section{EXPERIMENT AND ANALYSIS}

The schematic description of our structures is shown in Fig. 1. All four metallic (aluminum) structures have a subwavelength hole in the center with a diameter (a) of 8 $\mathrm{mm}$. The gratingless sample (Sample 1) with a thickness $(t)$ of $8 \mathrm{~mm}$ was used as a reference sample. The second sample has an identical aperture surrounded by six rectangular grooves. The periodicity of the grooves is $16 \mathrm{~mm}$, and the thickness $(w)$ of the grooved metal is $3.2 \mathrm{~mm}$. The measurements were performed in the microwave spectrum of $10-18 \mathrm{GHz}$, corresponding to a wavelength region of 16.7-30.0 mm. Figure 2 illustrates the experimental setup for the transmission and angular-distribution measurements. The experimental setup consists of an Hewlett Pockard 8510C network analyzer and two standard-gain horn antennas to measure the transmission amplitude. Radiation is normally incident upon the sample from $15 \mathrm{~cm}$ by the source antenna. The receiver antenna is $10 \mathrm{~cm}$ away from the sample for the transmission measurements.

For Sample 2, a transmission peak was measured around $13 \mathrm{GHz}$ with a transmission amplitude of 0.025 [Fig. 3(a)]. A twentyfold enhancement was achieved around the SP resonance frequency with Sample 2. For a further increase in transmission, we designed an annular aperture that can support a TE mode around $13 \mathrm{GHz}$. This structure (Sample 3) is identical to Sample 1, with a 

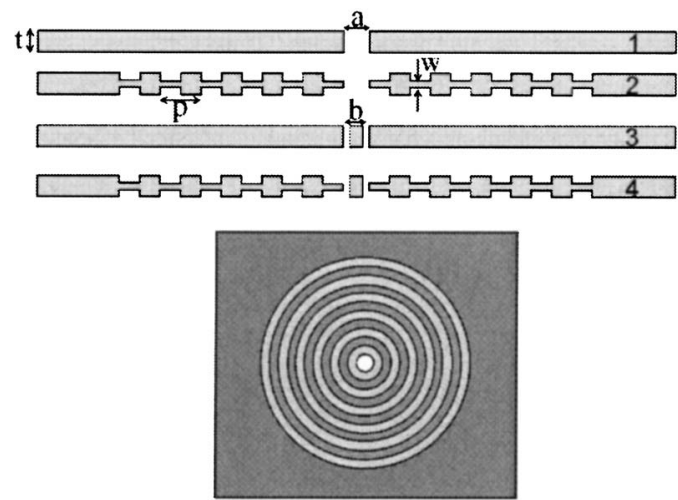

Fig. 1. Schematics of the four metallic samples and a top view of Sample 2.

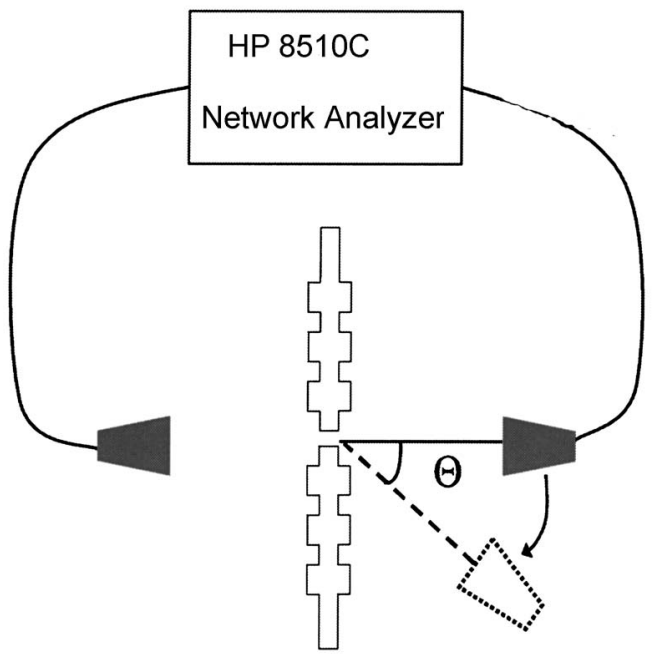

Fig. 2. Experiment setup for transmission and angular distribution measurements.

rod inside the hole. The diameter of the rod is $6.6 \mathrm{~mm}$, and the length of the rod is $8 \mathrm{~mm}$. The combination of the annular aperture and grooved structure (Sample 4) showed extraordinary high transmission at $12.9 \mathrm{GHz}$ $(23.25 \mathrm{~mm})$ via the coupling to the surface waves and the guided mode of annular aperture [Fig. 3(b)]. Including the reduction in the area of the aperture, a 450 -fold enhancement was achieved through the subwavelength annular aperture. $^{17}$

Enhancement of the transmission and beaming are referred to the SP waves on the surface. To observe these surface waves, we scanned the output surface of Sample 2. Owing to the restriction of the experimental setup, we were able to scan $4 \mathrm{~mm}$ away from the surface. Figure 4(a) shows the calculated field on the output surface of Sample 2, where the dotted curves refer to the aperture surface. The measured and calculated field was $4 \mathrm{~mm}$ away from the output surface of Sample 2 and is shown in Fig. 4(b). The FWHM of the measured field is equal to $9(\simeq \lambda / 2.5) \mathrm{mm}$. The subwavelength FWHM suggests the existence of the evanescent field at this distance.

In the experimental setup for angular-distribution measurements, the receiver antenna was placed $50 \mathrm{~cm}$ away from the sample's back face and was connected to a

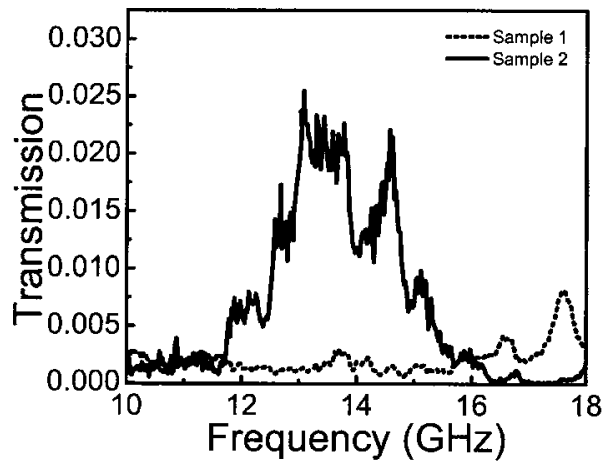

(a)

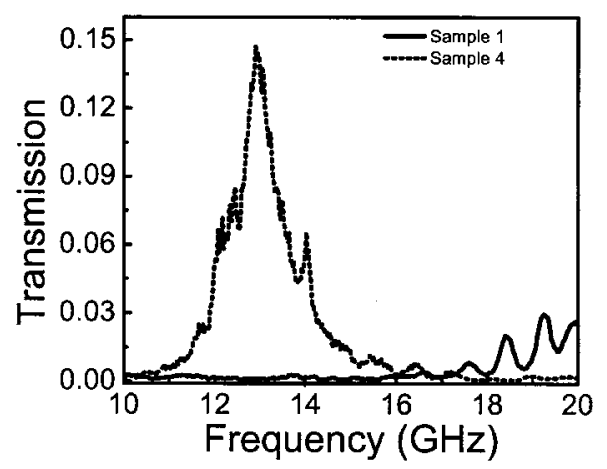

(b)

Fig. 3. Measured transmission results for (a) Samples 1 and 2 and (b) Samples 1 and 4.

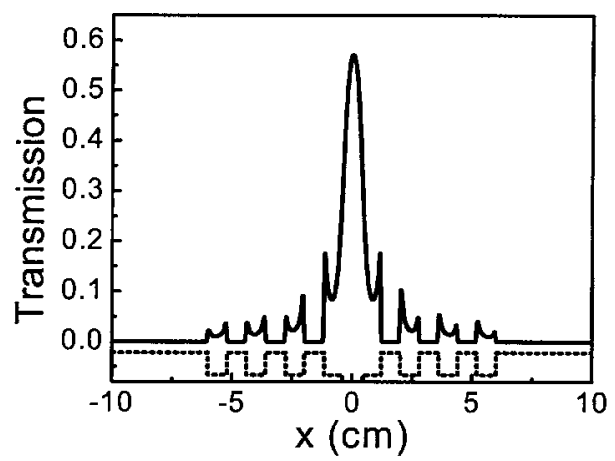

(a)

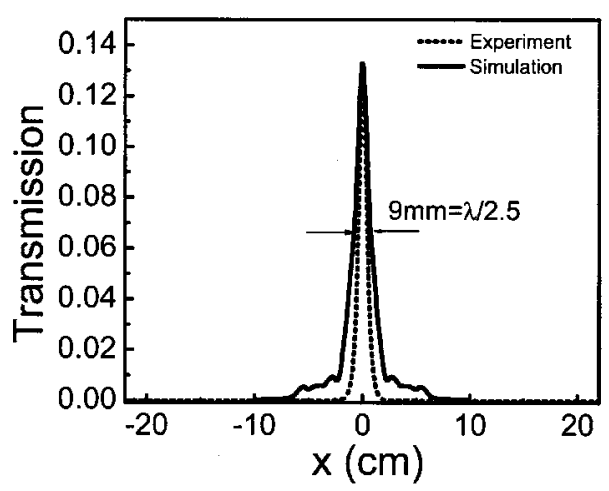

(b)

Fig. 4. (a) Calculated field on the output surface of Sample 2. (b) Measured and calculated field $4 \mathrm{~mm}$ away from the surface of Sample 2. 


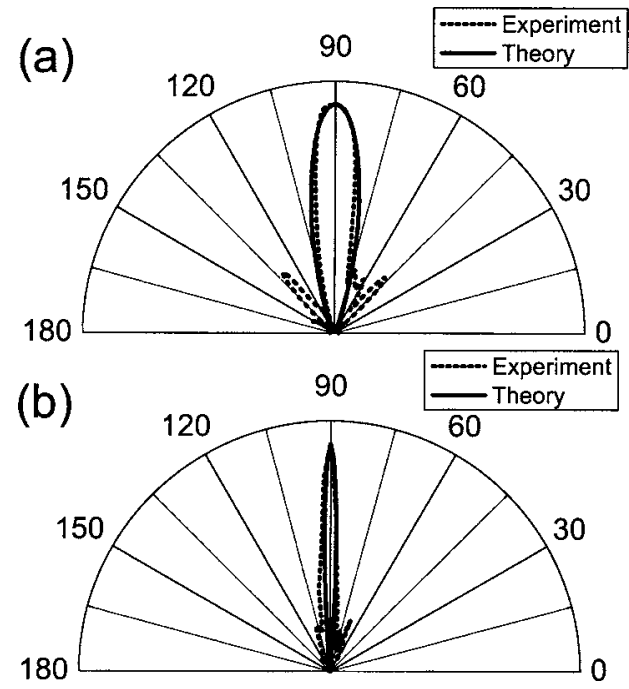

Fig. 5. Normalized angular transmission distributions for (a) Sample 1 and (b) Sample 2 at resonance frequency $(13 \mathrm{GHz})$.

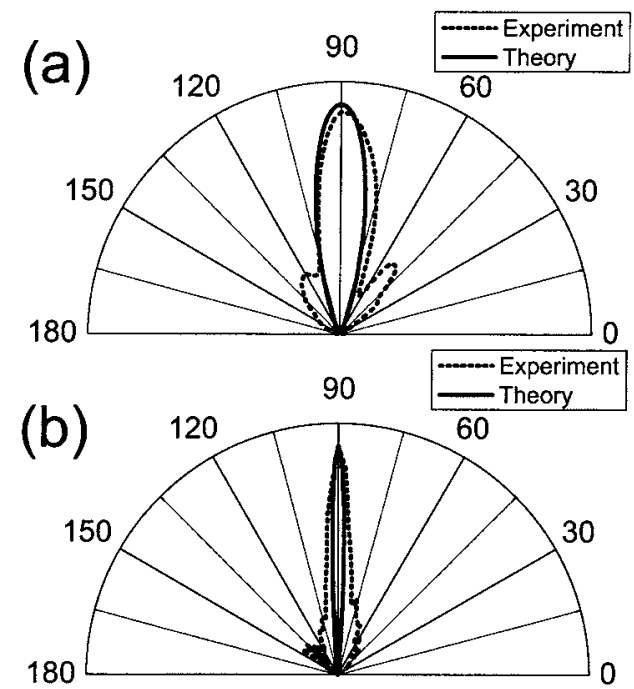

Fig. 6. Normalized angular transmission distributions for (a) Sample 3 and (b) Sample 4 at resonance frequency (13 GHz).

rotating arm to measure the angular dependence of the far-field radiation. Theoretical calculations were performed using simulations based on the finite-difference time-domain (FDTD) method based simulations.

Figure 5 shows the normalized measured and calculated angular transmission distribution at the resonance frequency for Samples 1 and 2. Angular divergence of the transmitted beam from Sample 1 is $\pm 12^{\circ}$, whereas the transmitted beam that emerges from Sample 2 is $\pm 3^{\circ}$. The angular divergence of the beam reduced 4 times compared with the transmitted beam from Sample 1.

The angular transmission intensity distribution at the enhanced transmission frequency for Samples 3 and 4 is presented in Fig. 6. FWHM divergence of the beam is $\pm 12^{\circ}$ and $\pm 3^{\circ}$, respectively. The angular divergence of the beam transmitted from Sample 3 is very similar to the beam transmitted through Sample 1.

The light emerging from the groove structure is emitted through a subwavelength aperture. It is expected that the emitted EM waves would quickly diffract in all direc- tions owing to the subwavelength dimensions of the aperture. ${ }^{1}$ On the contrary, we observed that the emitted EM waves are confined to a narrow spatial region when the subwavelength aperture was surrounded by periodic circular grooves. The surface-wave momentum and the momentum of the corrugation around the subwavelength aperture limit the allowed range of momentum of the reradiated EM waves. The circular symmetry of the structure suggests that the off-axis beams are suppressed owing to the destructive interference. In addition, the beams normal to the surface of the aperture constructively interfere, since the beams emitted from the hole and grooves are in phase.

The comparison of the calculated far-field distributions of EM waves emitted from Samples 1 and 4 is presented in Fig. 7. Calculated FWHM divergence of the beam transmitted from Samples 1 and 4 is $\pm 12^{\circ}$ and $\pm 3^{\circ}$, respectively, in agreement with measurements. Sample 4 can optimize the angular divergence of the beam, since FWHM of the beam is reduced fourfold compared with the beam transmitted through a subwavelength aperture (Sample 1).

Figure 8 shows the calculated (top) and measured (bottom) electric-field distribution of the transmitted beam from Sample 4 at the resonance frequency. The measured electric-field intensity at $13 \mathrm{GHz}$ over a region of a $44 \mathrm{~cm} \times 70 \mathrm{~cm}$ area on the output side of the aperture is measured with a monopole antenna with a resolution of $0.5 \mathrm{~cm}$. Our experiment shows that the electric-field in-

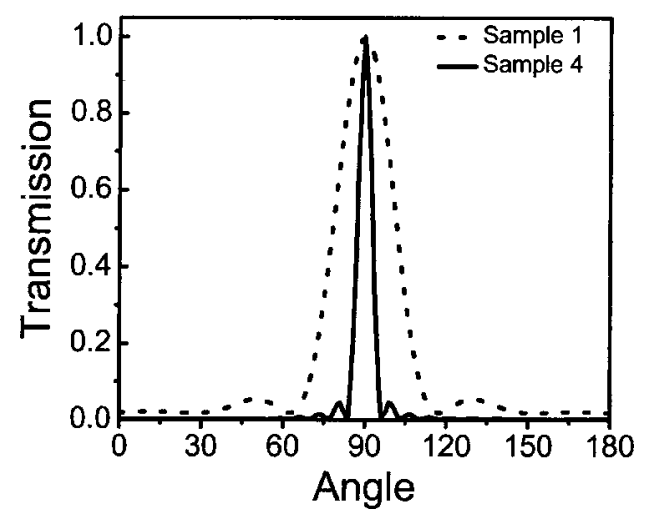

Fig. 7. Comparison of the calculated far-field results for Samples 1 and 4.

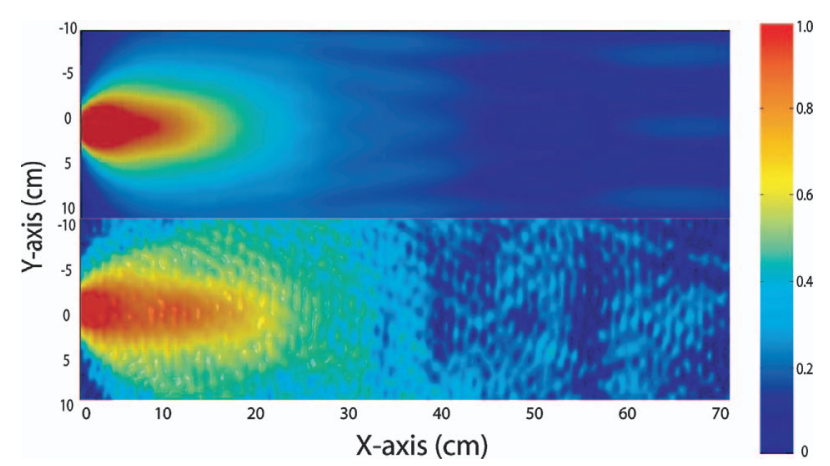

Fig. 8. Calculated (top) and measured (bottom) electric-field distribution from Sample 4 at the resonance frequency. 
tensity is confined to a narrow spatial region and propagates without diffracting into a wide angular region. The theoretical result is in good agreement with the experimental result.

\section{CONCLUSION}

In conclusion, we presented measured and calculated near-field and far-field EM distributions for subwavelength circular annular apertures with and without concentric periodic grooves in the microwave regime. We showed that the beam transmitted from circular apertures surrounded by concentric periodic grooves has a subwavelength FWHM near the aperture. Furthermore, we measured the normalized angular transmission distributions from these apertures. The beam transmitted from the circular annular apertures surrounded by concentric grooves (Sample 4) is 450 times more enhanced, and the angular divergence of the beam is fourfold reduced compared with the beam transmitted through a subwavelength aperture (Sample 1). These effects can be explained by coupling of EM waves to the SPs and the guided mode of annular aperture. The experimental results are in good agreement with FDTD-based theoretical calculations.

\section{ACKNOWLEDGMENTS}

This work was supported by projects EU-DALHM, EU NOE-METAMORPHOSE, EU NOE-PHOREMOST, and TUBITAK-104E090. One of the authors (E.O.) acknowledges partial support from the Turkish Academy of Sciences.

$\mathrm{H}$. Caglayan is the corresponding author and can be reached via e-mail at caglayan@fen.bilkent.edu.tr.

\section{REFERENCES}

1. H. A. Bethe, "Theory of diffraction by small holes," Phys. Rev. 66, 163182 (1944).

2. H. J. Lezec, A. Degiron, E. Devaux, R. A. Linke, L. MartinMoreno, F. J. Garcia-Vidal, and T. W. Ebbesen, "Beaming light from a subwavelength aperture," Science 297, $820-822(2002)$

3. F. J. Garcia-Vidal, H. J. Lezec, T. W. Ebbesen, and L. Martin-Moreno, "Multiple paths to enhance optical transmission through a single subwavelength slit," Phys. Rev. Lett. 90, 213901 (2003).

4. S. S. Akarca-Biyikli, I. Bulu, and E. Ozbay, "Enhanced transmission of microwave radiation in one-dimensional metallic gratings with subwavelength aperture," Appl. Phys. Lett. 85, 1098-1100 (2004).

5. L. Martin-Moreno, F. J. Garcia-Vidal, H. J. Lezec, A Degiron, and T. W. Ebbesen, "Theory of highly directional emission from a single subwavelength aperture surrounded by surface corrugations," Phys. Rev. Lett. 90, 167401 (2003).

6. M. J. Lockyear, A. P. Hibbins, J. R. Sambles, and C. R. Lawrence, "Surface-topography-induced enhanced transmission and directivity of microwave radiation through a subwavelength circular metal aperture," Appl. Phys. Lett. 84, 2040-2042 (2004).

7. J. M. Steele, C. E. Moran, A. Lee, C. M. Aguirre, and N. J. Halas, "Metallodielectric gratings with subwavelength slots: optical properties," Phys. Rev. B 68, 205103 (2003).

8. N. Bonod, S. Enoch, L. Li, P. Evgeny, and M. Neviere, "Resonant optical transmission through thin metallic films with and without holes," Opt. Express 11, 482-490 (2003).

9. A. G. Borisov, F. J. Garca de Abajo, and S. V. Shabanov, "Role of electromagnetic trapped modes in extraordinary transmission in nanostructured materials," Phys. Rev. B 71, 075408 (2005).

10. S. S. Akarca-Biyikli, I. Bulu, and E. Ozbay, "Resonant excitation of surface plasmons in one-dimensional metallic grating structures at microwave frequencies," J. Opt. A Pure Appl. Opt. 7, 159-164 (2005).

11. M. J. Lockyear, A. P. Hibbins, J R. Sambles, and C. R Lawrence, "Enhanced microwave transmission through a single subwavelength aperture surrounded by concentric grooves," J. Opt. A Pure Appl. Opt. 7, 152-158 (2005).

12. H. Raether, Surface Plasmons on Smooth and Rough Surfaces and on Gratings (Springer-Verlag, 1988).

13. Y. Teng, E. A. Stern, "Plasma radiation from metal grating surfaces," Phys. Rev. Lett. 19, 511-514 (1967).

14. T. Thio, H. J. Lezec, T. W. Ebessen, K. M. Pellerin, G. D. Lewen, A. Nahata, and R. A. Linke, "Giant optical transmission of sub-wavelength apertures: physics and applications," Nanotechnology 13, 429-432 (2002).

15. U. C. Fischer and M. Zapletal, "The concept of a coaxial tip as a probe for scanning near field optical microscopy and steps towards a realisation," Ultramicroscopy 42-44, 393-398 (1992).

16. F. I. Baida, D. Van Labeke, and B. Guzial, "Enhanced confined light transmission by single subwavelength apertures in metallic films," Appl. Opt. 42, 6811-6815 (2003).

17. H. Caglayan, I. Bulu, and E. Ozbay, "Extraordinary grating-coupled microwave transmission through a subwavelength annular aperture," Opt. Express 13, 1666-1671 (2005). 